\title{
Estimación del sobrediagnóstico de cáncer mamario luego de la implementación de un programa de rastreo
}

Estimate of overdiagnosis of breast cancer after implementation of a screening program

\section{Objetivo}

Estimar la tasa de sobrediagnóstico de cáncer mamario (CM) atribuible a su rastreo poblacional y determinar el cambio en la incidencia de $\mathrm{CM}$ invasivo e intraductal luego de la implementación masiva de un programa de rastreo.

\section{Métodos}

En 1988 se implementó en Columbia Británica, Canadá, un programa de rastreo de $\mathrm{CM}$ en mujeres de 40 a 89 años de edad que alcanzó una cobertura del $100 \%$ en 2000 . A través de los registros de Cáncer de Mama y de dicho Programa de Rastreo, se comparó la incidencia de CM entre dos períodos: "1970 a 1979" (época en la que el rastreo era escaso y mal estructurado), "2005 a 2009" (años en los que los programas de rastreo ya estaban bien establecidos).

Durante este último periodo, las mujeres fueron clasificadas en dos grupos: las que habían realizado una mamografía anual durante los cinco años previos (rastreo periódico); las que no habían cumplido con los criterios de rastreo activo (rastreo esporádico) $)^{\ddagger}$.

\section{Medición de resultados principales}

Fueron estimadas las incidencias de CM invasivo e intraductal en ambos períodos (1970-1979 y 2005-2009) y sus incidencias relativas. Para estimar la tasa de sobrediagnóstico fueron calculadas las tasas de incidencia específicas por edad de CM invasivo e intraductal utilizando dos métodos: a) la comparación de datos individuales de grupos con diferente intensidad de rastreo durante el mismo período (2005 a 2009); b) la comparación de datos agregados entre los dos períodos (antes y después de la diseminación del masivo) respecto de la incidencia de $\mathrm{CM}$ intraductal e invasivo.

\section{Resultados}

La incidencia acumulativa de CM invasivo en el subgrupo que realizó rastreo esporádico entre los 40 y los 89 años fue de 130 casos cada mil mujeres y la del grupo rastreado en forma periódica, 137 cada mil; lo que implica un aumento absoluto en el diagnóstico de $\mathrm{CM}$ invasivo atribuible a la intensidad del rastreo de siete casos cada mil mujeres. Como era esperable, se observó un aumento de la incidencia de CM intraductal entre 2005 y 2009 (atribuible al rastreo) cuando se la comparó con la documentada entre 1970 y 1979. Ver tabla 1.

Tabla 1: incidencia relativa de cáncer mamario invasivo e intraductal por rango etario entre los periodos "2005 a 2009" y "1970 a 1979" (después y antes de la implementación del programa de rastreo mamográfico).

\begin{tabular}{c|c|c}
\multirow{2}{*}{$\begin{array}{c}\text { Edad } \\
\text { en años }\end{array}$} & \multicolumn{2}{|c}{$\begin{array}{c}\text { Incidencias relativas (después/antes) de la } \\
\text { diseminación del programa de rastreo (IC95\%) }\end{array}$} \\
\cline { 2 - 3 } $\mathbf{3 0}$ a 39 & Carcinoma intraductal & Carcinoma invasivo \\
\hline $\mathbf{4 0}$ a 49 & $2,02(1,27$ a 3,23$)$ & $0,92(0,82$ a 1,04$)$ \\
\hline $\mathbf{5 0}$ a $\mathbf{5 9}$ & $4,10(3,31$ a 5,08$)$ & $1,01(0,95$ a 1,07$)$ \\
\hline $\mathbf{6 0}$ a $\mathbf{6 9}$ & $4,64(3,80$ a 5,67$)$ & $0,97(0,92$ a 1,02$)$ \\
\hline $\mathbf{7 0}$ a $\mathbf{7 9}$ & $7,49(5,87$ a 9,57$)$ & $1,21(1,15$ a 1,28$)$ \\
\hline Más de $\mathbf{8 0}$ & $6,43(4,79$ a 8,62$)$ & $1,25(1,18$ a 1,33$)$ \\
\hline & $1,40(0,95$ a 2,05$)$ & $1,06(0,98$ a 1,15$)$ \\
\hline
\end{tabular}

Sin embargo y contrariamente a lo esperado, no se observó declinación en la incidencia de CM invasivo y sí, por el contrario, un incremento de la misma entre los 60 y los 79 años.

\section{Conclusiones}

Independientemente de la periodicidad de la pesquisa, no se observó una disminución de la incidencia de CM invasivo luego de la diseminación de un programa de rastreo masivo de CM.

Fuente de financiamiento: no referida. Conflictos de interés de los autores: A.C. había trabajado en el Programa de Rastreo con mamografía de la Columbia Británica (CB). Ambos autores son actualmente empleados de la Agencia de Cáncer de la CB.

\section{Comentario}

Se denomina sobrediagnóstico al correcto diagnóstico de una condición que no habría causado daño ni muerte, y que que en el caso del CM conlleva riesgos para la paciente debido a la cascada diagnóstica y terapéutica que acarrea su detección. En este contexto, la historia natural del $\mathrm{CM}$ se encuentra actualmente en revisión ya que muchos cánceres detectados por mamografía (aún los de patrón histológico invasivo y más aún los de patrón intraductal) podrían regresar en forma espontánea'.

Los autores de la investigación documentaron el sobrediagnóstico en términos del "exceso" de cánceres detectados luego de la diseminación masiva un los programas de rastreo de CM. Estos datos nos permiten suponer que este aumento de la incidencia CM atribuible a la implementación del rastreo masivo fue a costa de la detección formas más precoces y de mejor pronóstico que las que suelen ser detectadas en etapas sin- tomáticas. Si esta detección "temprana" tuviera impacto en la historia natural de la enfermedad, debería haberse acompañado de una disminución de los diagnósticos de CM invasivo, lo que no pudo ser documentado en este trabajo poblacional. Esto nos lleva a pensar que la mayoría de los CM detectados a través del programa de rastreo fueron formas indolentes que habrían evolucionado en forma probablemente favorable.

\section{Conclusiones de la comentadora}

El riesgo de sobrediagnóstico al implementar programas de rastreo mamográfico del $\mathrm{CM}$ es significativo y aún no queda claro el balance entre los riesgos y los beneficios de esta práctica preventiva cada vez más cuestionada.

María Victoria Salgado [ Servicio de Medicina Familiar y Comunitaria del Hospital Italiano de Buenos Aires maria.salgado@ hospitalitaliano.org.ar ]

Salgado M. Estimación del sobrediagnóstico de cáncer mamario luego de la implementación de un programa de rastreo. Evid Act Pract Ambul. 2014:17(3).Jul-Sep. 86. Comentado de: Coldman A. et al. Incidence of breast cancer and estimates of overdiagnosis after the initiation of a population-based mammography screening program. CMAJ. 2013;185(10):E492-8. PMID: 23754101.

Referencia

1. Zahl P y col. Natural history of breast cancers detected in the Swedish mammography screening programme: a cohort study. The lancet oncology. 2011;12(12):1118-24. 